9. Future X-ray Observatories, Detectors and Instrumentation 


\title{
A HIGH RESOLUTION ECHELLE SPECTROMETER FOR SOFT X-RAY AND EUV ASTRONOMY
}

\author{
James Green and Stuart Bowyer \\ Space Sciences Laboratory, University of California, Berkeley, CA, USA
}

\begin{abstract}
We present a new design for high resolution spectroscopy from 80 to $400 \AA$. This design employs grazing incidence optics and variable line-spaced gratings to achieve high resolution. Unlike some previously proposed EUV echelles, this design employs straight groove planar gratings, which are a well-proven, easily manufactured design. The instrument delivers a peak resolution of $\lambda \Delta \lambda=7500$ and a peak effective area of $3 \mathrm{~cm}^{2}$.
\end{abstract}

\section{INTRODUCTION}

High resolution spectroscopy shortward of the IUE cutoff is a high priority for the next generation of space instruments. The Far Ultraviolet Spectroscopic Explorer will give high resolution down to $900 \AA$. The Orbiting Retrievable Far and Extreme Ultraviolet Spectrometer will give resolution in excess of 5000 down to $400 \AA$. Therefore, we have designed this instrument to provide resolution on the order of 5000 with a wavelength coverage for $80-400$ $\AA$. The instrument has been designed with a 1 -m diameter optic, although the concept is also valid for smaller systems. We discuss the tradeoffs resulting from a reduction in aperture.

\section{MIRROR DESIGN}

For high efficiency at the wavelengths of operation, it is necessary to employ a grazing incidence telescope. Additionally, optical systems in this wavelength region are highly susceptible to "contamination" from diffuse emission. In this particular application, problems could arise from diffuse emission at 304 and $1216 \AA$. Even though $1216 \AA$ is outside the instrument bandpass, the diffuse emissions are so intense that the scattered intensity from the grating can be larger than the signal if the instrument is not properly baffled. Fortunately, one grazing incidence optical design allows strict control of off-axis light (Green and Bowyer 1986). This is the Hettrick-Bowyer Type I design, which is a solution to the coma-free Schwarzschild equations. The primary mirror is a slightly modified paraboloid, and the secondary mirror is a slightly modified ellipsoid. The light comes to a focus between the mirrors. This design represents the grazing incidence analogue to a Gregorian telescope, just as a WolterSchwarzschild Type II represents the grazing incidence analogue to a Cassegrain. By placing an aperture at this primary focus, the field of view and, therefore, the diffuse emission are strictly controlled. A few additional baffles are all that is needed to eliminate problems from diffuse emission. The mirror chosen for this experiment is an $\mathrm{f} / 10$ optic with a 3-m front-tofocus length. The mirror parameters are presented in Table 1.

\section{THE GRATINGS}

Three sets of two gratings intercept the beam directly after the secondary mirror. The first grating operates in a high order and acts as the echelle; the second grating operates in first order and acts as a cross disperser. Each echelle grating intercepts $1 / 8$ of the total beam. Since we have chosen to use straight groove variable line-spaced gratings, the resolution is inherently limited by the speed of the beam accepted by the grating in the direction perpendicular to the dispersion (Hettrick 1984). In order to achieve our resolution goal, the grating could not be any wider than one which intercepted $1 / 8$ of the beam. If the grating width were increased, resolution would decrease, but effective area would increase. The echelle line 
Table 1. Instrument Parameters

\begin{tabular}{|ll|}
\hline Mirror & Hettrick-Bowyer Type I \\
Diameter & 1 meter \\
Focal Length & 3 meters \\
Gratings (6) & Varied line-spaced, planar \\
Detectors & Microchannel plate, \\
& Wedge and strip anode \\
Resolution & $40 \mu$ \\
Photocathode & $\mathrm{KBr}$ \\
Instrument Performance & \\
Coverage & $80-400 \AA$ \\
Resolution & $>5000$ \\
Average effective area & $\sim 1 \mathrm{~cm}^{2}$ \\
\hline
\end{tabular}

spacing is optimized at one $m \lambda$, where $m$ is the order number and $\lambda$ the wavelength.

The cross disperser is oriented so that the beam intersects the grating normal to the ruled direction (standard in-plane mount). This allows the line spacing to be optimized again for one wavelength. The gratings designed in this manner are quite straightforward to manufacture. Their parameters are listed in Table 1.

\section{THE DETECTORS}

The detectors employed are microchannel plate detectors with wedge and strip anodes (Martin et al. 1981). The system has been designed for detectors with a $25-\mathrm{mm}$ active area. In all performance calculations we have assumed that a potassium bromide photocathode will be applied (Siegmund et al. 1988). To achieve the spectral resolution stated, it is necessary that the detector have a resolution of 40 microns. This is a reasonable expectation for a $25-$ mm detector.

\section{SYSTEM PERFORMANCE}

An assembly drawing of the instrument is provided in Fig. 1. For clarity, only one of the three echelle/cross disperser grating sets is shown. A direct imaging detector is placed at the telescope focus, allowing tracking of the target and compensation for drift for maximum resolution. The system's effective area was calculated by determining the reflectivity of each optical component, assuming the use of a gold coating and incorporating the effects of polarization. The grating efficiency was calculated with a standard diffraction efficiency code that includes shadowing effects. Since the gratings operate near the blazed $m \lambda$ at nearly all $\lambda$, the diffraction efficiency remains high, and groove shadowing becomes the dominant limiting factor. We assumed the detector quantum efficiency matched the experimental values for potassium bromide as measured in our laboratory.

The resolution of the instrument was verified by raytrace analyses of the entire system. Randomly placed parallel rays were raytraced through the mirror and then given a random three-arcsecond blur simulating the intrinsic blur in the mirror caused by small figure errors. The rays were then traced through both gratings to the detector plane. The resulting images are displayed in Figs. 2 and 3. It can be seen that the absolute resolution of the system (dispersion/image width) is in excess of 7500 and that the FWHM resolution is even higher, $\sim$ 10,000. These instrument performance characteristics are also included in Table 1. 


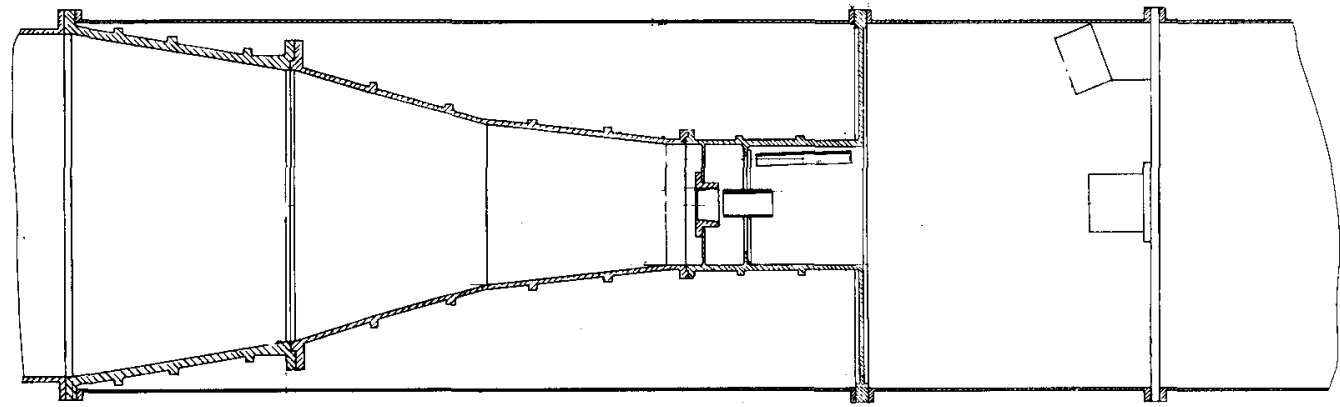

3 meters

Figure 1. A schematic drawing of the instrument assembly. The primary and secondary mirrors are joined by an aluminum metering structure that is precision-machined to maintain the correct mirror alignment. For clarity, only two of the six gratings are shown, along with the detector. The detector at the mirror focus performs direct imaging and drift correction.

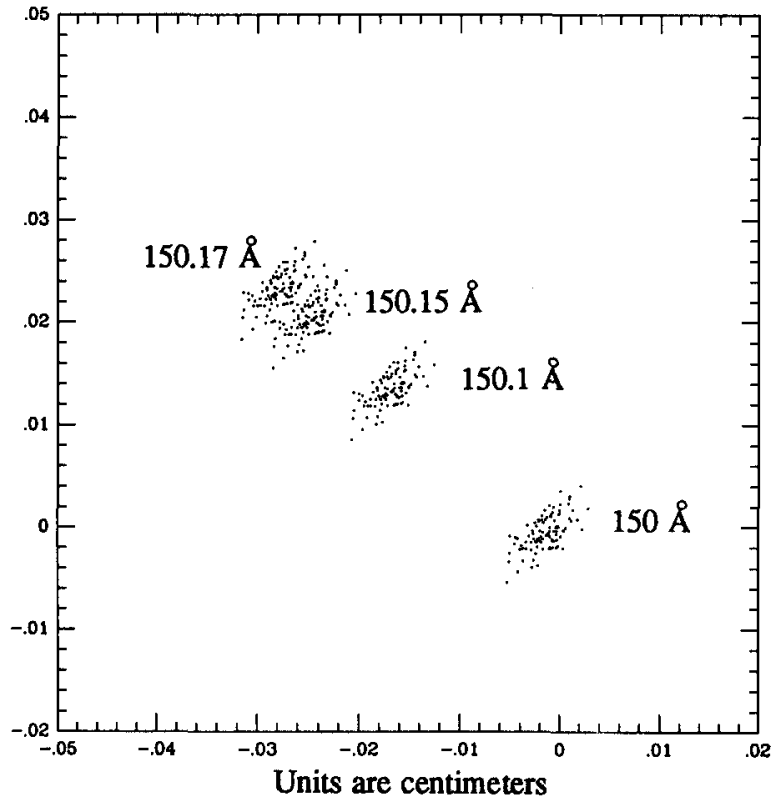

Figure 2. A raytrace result demonstrating the resolution achieved by this instrument. The raytraces include a three-arcsecond blur from the optics. Clearly, with $40 \mu$ detector resolution, $0.02 \AA$ at $150 \AA$ can be resolved for a resolution of 7500 . With better detectors, a FWHM resolution in excess of 10,000 can be achieved. 


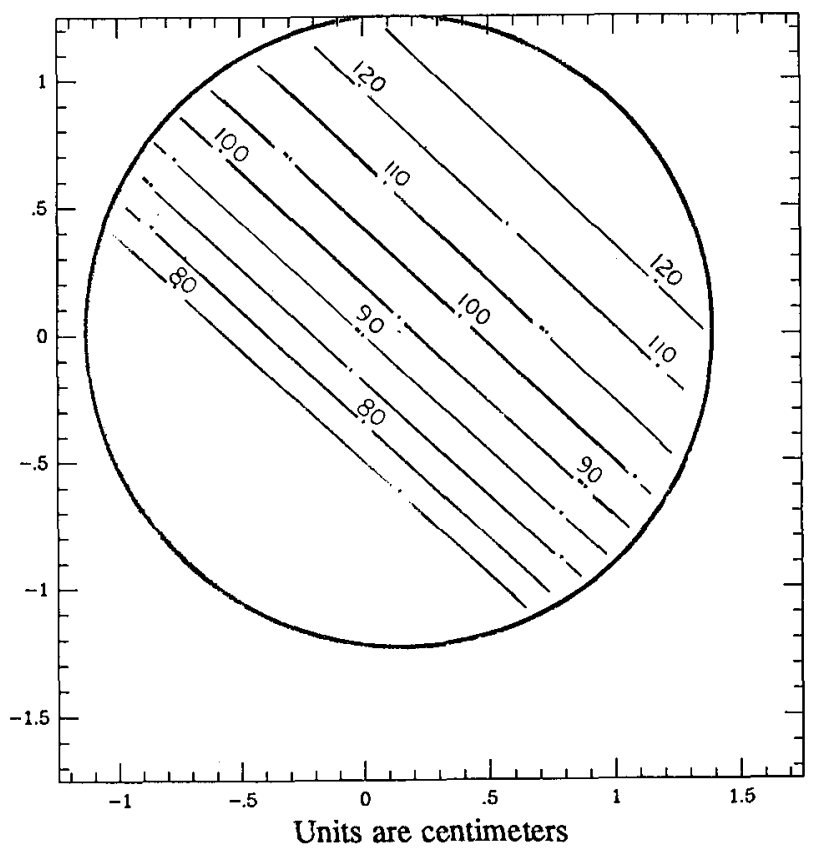

Figure 3. This raytrace result demonstrates coverage of the echelles from 80 to $120 \AA$. The lines drawn show the various orders, and the small dots are the actual image sizes. Several wavelengths are labeled for identification. Note that each wavelength appears in at least two orders. The circle drawn represents the active area of the detector.

When a similar design is incorporated into a smaller package, instrument performance is not degraded significantly. Since each grating sees only one section of the mirror aperture, the effective area goes linearly with mirror radius, not as the square. Also, as the mirror radius decreases, if the length is maintained, the graze angles on the mirror decrease, and the reflectivity increases. This is especially true at shorter wavelengths. For example, if the instrument diameter is reduced to $50 \mathrm{~cm}$, fully $75 \%$ of the $1-\mathrm{m}$ diameter instrument average effective area can be achieved.

\section{ACKNOWLEDGMENTS}

This work was supported by NASA grant NGR-05-003-450.

\section{REFERENCES}

Green, J., and Bowyer, S. 1986, Appl. Opt., 25, 1991.

Hettrick, M. C. 1984, Appl. Opt., 23, 3221.

Martin, C., Jelinsky, P., Lampton, M., Malina, R. F., and Anger, H. O. 1981, Rev. Sci. Instrum., 52, 1967.

Siegmund, O. H. W., Everman, E., Vallerga, J. V., and Lampton, M. 1988, Appl. Opt., 27, 1568. 